International Mathematical Forum, 2, 2007, no. 56, 2771 - 2779

\title{
An Unconditionally A-Stable Method for Initial Value Problems Based on Simpson's Rule ${ }^{1}$
}

\author{
Juan C. Aguilar \\ Intituto Tecnológico Autónomo de México (ITAM) \\ Departamento de Matemáticas, México, D.F. 01000, Mexico \\ aguilar@itam.mx
}

\begin{abstract}
In this paper we construct an algorithm to approximate the solution of the initial value problem $y^{\prime}(t)=f(t, y)$ with $y\left(t_{0}\right)=y_{0}$. The method is implicit and combines the classical Simpson's rule with the Simpson's $3 / 8$ rule to yield an unconditionally A-stable method of order 4.
\end{abstract}

Mathematics Subject Classification: 65L05

Keywords: Initial value problem, quadratures, Simpson's rule, A-stability

\section{Introduction}

In this work we describe a numerical method based on Simpson's rule to approximate the solution of the initial value problem $y^{\prime}(t)=f(t, y)$, where $f: \mathbb{R}^{2} \rightarrow \mathbb{R}$ is a function of class $C^{1}$. As is well known, Simpson's rule has been used in numerical methods for the solution of initial value problems, (see [2], [3], [4], [6]). All of these methods are conditionally A-stable, requiring small steps for stiff problems in order to yield accurate approximations.

The method we propose in this paper combines Simpson's rule with Simpson's $3 / 8$ rule to obtain an unconditionally $A$-stable algorithm. The procedure is not a multistep method, and differs from most one-step methods in the sense that it simultaneously yields 3 approximations of $y(t)$ at $t=t_{0}+h, t_{0}+2 h$, and $t_{0}+3 h$ from the value $y\left(t_{0}\right)$. To accomplish 4 th-order accuracy and A-stability, the algorithm solves a 3 by 3 system of algebraic equations.

\footnotetext{
${ }^{1}$ This works was supported by Asociación Mexicana de Cultura A.C.
} 
Simpson's 3/8 rule has been used in combination with Simpson's rule by Milne and Reynolds (see [3]) in order to improve the stability properties of a method proposed by Milne in [2].

\section{Integration of $y^{\prime}(t)=f(t, y)$ with Simpson's rule}

In this section we describe how Simpson's rule and the Simpson's 3/8 rule are combined to approximate the solution of the initial value problem

$$
\begin{aligned}
y^{\prime}(t) & =f(t, y(t)), \quad t \in\left[t_{0}, b\right] \\
y\left(t_{0}\right) & =y_{0} .
\end{aligned}
$$

The classical Simpson's rule approximates a definite integral of a function $g$ as follows: let $t_{0}<t_{1}<t_{2}$ be three equally spaced points, and let $h=t_{1}-t_{0}$. Then

$$
\int_{t_{0}}^{t_{2}} g(t) d t \approx \frac{h}{3}\left[g\left(t_{0}\right)+4 g\left(t_{1}\right)+g\left(t_{2}\right)\right] .
$$

Simpson's 3/8 rule approximates a definite integral of $g$ requiring four equally spaced points: let $t_{0}<t_{1}<t_{2}<t_{3}$ be equally spaced, with $h=t_{1}-t_{0}$. The approximation takes the form

$$
\int_{t_{0}}^{t_{3}} g(t) d t \approx \frac{3 h}{8}\left[g\left(t_{0}\right)+3 g\left(t_{1}\right)+3 g\left(t_{2}\right)+g\left(t_{3}\right)\right] .
$$

Both integration rules have an approximation error proportional to $h^{5}$ provided $g$ is of class $C^{4}$ (see [7]). We now describe how this rules are used to discretize (1).

Let $t_{0}<t_{1}<t_{2}<t_{3}$ be a regular partition of an interval $\left[t_{0}, t_{0}+T_{0}\right]$, hence $t_{k}=t_{0}+k h$ with $h=T_{0} / 3$. Integrating both sides of $(1)$ on $\left[t_{0}, t_{2}\right]$ we obtain

$$
y\left(t_{2}\right)-y\left(t_{0}\right)=\int_{t_{0}}^{t_{2}} f(t, y(t)) d t
$$

Applying Simpson's rule (2) to (4) we get the approximation

$$
y\left(t_{2}\right)-y\left(t_{0}\right) \approx \frac{h}{3}\left(f\left(t_{0}, y\left(t_{0}\right)\right)+4 f\left(t_{1}, y\left(t_{1}\right)\right)+f\left(t_{2}, y\left(t_{2}\right)\right)\right) .
$$


Similarly, integrating both sides of $(1)$ on $\left[t_{1}, t_{3}\right]$ and approximating the integral with Simpson's rule we obtain

$$
y\left(t_{3}\right)-y\left(t_{1}\right) \approx \frac{h}{3}\left(f\left(t_{1}, y\left(t_{1}\right)\right)+4 f\left(t_{2}, y\left(t_{2}\right)\right)+f\left(t_{3}, y\left(t_{3}\right)\right)\right) .
$$

Integrating now both sides of (1) on $\left[t_{0}, t_{3}\right]$ and using Simpson's $3 / 8$ rule to approximate the integral,

$$
\left.y\left(t_{3}\right)-y\left(t_{0}\right) \approx \frac{3 h}{8}\left(f\left(t_{0}, y\left(t_{0}\right)\right)+3 f\left(t_{1}, y\left(t_{1}\right)\right)+3 f\left(t_{2}, y\left(t_{2}\right)\right)+f\left(t_{3}, y\left(t_{3}\right)\right)\right)\right) .
$$

Given the initial value $y_{0}=y\left(t_{0}\right)$, to obtain approximations $y_{1}$ to $y\left(t_{1}\right), y_{2}$ to $y\left(t_{2}\right)$, and $y_{3}$ to $y\left(t_{3}\right)$, from (5)-(7) we set a system of three algebraic equations with the three unknowns $y_{1}, y_{2}, y_{3}$ :

$$
\begin{aligned}
y_{2}-y_{0} & =\frac{h}{3}\left(f\left(t_{0}, y_{0}\right)+4 f\left(t_{1}, y_{1}\right)+f\left(t_{2}, y_{2}\right)\right) \\
y_{3}-y_{1} & =\frac{h}{3}\left(f\left(t_{1}, y_{1}\right)+4 f\left(t_{2}, y_{2}\right)+f\left(t_{3}, y_{3}\right)\right) \\
y_{3}-y_{0} & =\frac{3 h}{8}\left(f\left(t_{0}, y_{0}\right)+3 f\left(t_{1}, y_{1}\right)+3 f\left(t_{2}, y_{2}\right)+f\left(t_{3}, y_{3}\right)\right) .
\end{aligned}
$$

The above system of equations can be written in the form $F_{h}\left(y_{1}, y_{2}, y_{3}\right)=$ $(0,0,0)$. The Jacobian matrix of $F_{h}$ is given by

$$
F_{h}^{\prime}=\left(\begin{array}{ccc}
-\frac{4 h}{3} \frac{\partial f}{\partial y_{1}}\left(t_{1}, y_{1}\right) & 1-\frac{h}{3} \frac{\partial f}{\partial y_{2}}\left(t_{2}, y_{2}\right) & 0 \\
-1-\frac{h}{3} \frac{\partial f}{\partial y_{1}}\left(t_{1}, y_{1}\right) & -\frac{4 h}{3} \frac{\partial f}{\partial y_{2}}\left(t_{2}, y_{2}\right) & 1-\frac{h}{3} \frac{\partial f}{\partial y_{3}}\left(t_{3}, y_{3}\right) \\
-\frac{9 h}{8} \frac{\partial f}{\partial y_{1}}\left(t_{1}, y_{1}\right) & -\frac{9 h}{8} \frac{\partial f}{\partial y_{2}}\left(t_{2}, y_{2}\right) & 1-\frac{3 h}{8} \frac{\partial f}{\partial y_{3}}\left(t_{3}, y_{3}\right)
\end{array}\right)
$$

Hence, $F_{h}^{\prime}=A+h B$, where

$$
A=\left(\begin{array}{ccc}
0 & 1 & 0 \\
-1 & 0 & 1 \\
0 & 0 & 1
\end{array}\right), \quad B=\left(\begin{array}{ccc}
-\frac{4}{3} \frac{\partial f}{\partial y_{1}}\left(t_{1}, y_{1}\right) & -\frac{1}{3} \frac{\partial f}{\partial y_{2}}\left(t_{2}, y_{2}\right) & 0 \\
-\frac{1}{3} \frac{\partial f}{\partial y_{1}}\left(t_{1}, y_{1}\right) & -\frac{4}{3} \frac{\partial f}{\partial y_{2}}\left(t_{2}, y_{2}\right) & -\frac{1}{3} \frac{\partial f}{\partial y_{3}}\left(t_{3}, y_{3}\right) \\
-\frac{9}{8} \frac{\partial f}{\partial y_{1}}\left(t_{1}, y_{1}\right) & -\frac{9}{8} \frac{\partial f}{\partial y_{2}}\left(t_{2}, y_{2}\right) & -\frac{3}{8} \frac{\partial f}{\partial y_{3}}\left(t_{3}, y_{3}\right)
\end{array}\right)
$$

If $f$ is of class $C^{1}$, and if $\left(t_{1}, t_{2}, t_{3}, y_{1}, y_{2}, y_{3}\right)$ is restricted to a bounded set in $\mathbb{R}^{6}$, there exists $\epsilon>0$ such that if $0<h<\epsilon$, then $F_{h}^{\prime}$ is invertible since $\mathrm{A}$ is invertible. By the inverse function theorem $F_{h}$ is locally invertible. Furthermore, we have the folowing result: 
Theorem 2.1 If $f$ is of class $C^{1}$, the system of algebraic equations (8)-(10) has a unique solution in a neighborhood of $\left(y_{0}, y_{0}, y_{0}\right)$ for $h$ sufficiently small.

\section{Proof}

Substracting equation (9) from (10) and reordering the equations, we obtain an equivalent system of the form

$$
\begin{aligned}
& y_{1}-y_{0}=h g_{1}\left(t_{0}, t_{1}, t_{2}, t_{3}, y_{0}, y_{1}, y_{2}, y_{3}\right) \\
& y_{2}-y_{0}=h g_{2}\left(t_{0}, t_{1}, t_{2}, t_{3}, y_{0}, y_{1}, y_{2}, y_{3}\right) \\
& y_{3}-y_{0}=h g_{3}\left(t_{0}, t_{1}, t_{2}, t_{3}, y_{0}, y_{1}, y_{2}, y_{3}\right)
\end{aligned}
$$

where $g_{1}=\left(3 f\left(t_{0}, y_{0}\right)+19 f\left(t_{1}, y_{1}\right)-5 f\left(t_{2}, y_{2}\right)+f\left(t_{3}, y_{3}\right)\right) / 24, g_{2}=\left(f\left(t_{0}, y_{0}\right)+\right.$ $\left.4 f\left(t_{1}, y_{1}\right)+f\left(t_{2}, y_{2}\right)\right) / 3$, and $g_{3}=3\left(f\left(t_{0}, y_{0}\right)+3 f\left(t_{1}, y_{1}\right)+3 f\left(t_{2}, y_{2}\right)+f\left(t_{3}, y_{3}\right)\right) / 8$. Here $g_{1}, g_{2}$ y $g_{3}$ are of class $C^{1}$ sice they are linear combinations of evaluations of $f$. This system of equations can be written in the form $\boldsymbol{y}-W_{h}(\boldsymbol{y})=\mathbf{0}$, with $\boldsymbol{y}=\left(y_{1}, y_{2}, y_{3}\right)$. The Jacobian matrix of $W_{h}$ is of the form $W_{h}^{\prime}(\boldsymbol{y})=h S(\boldsymbol{y})$ where

$$
S(\boldsymbol{y})=\left(\begin{array}{ccc}
\frac{19}{24} \frac{\partial f}{\partial y_{1}}\left(t_{1}, y_{1}\right) & -\frac{5}{24} \frac{\partial f}{\partial y_{2}}\left(t_{2}, y_{2}\right) & \frac{1}{24} \frac{\partial f}{\partial y_{3}}\left(t_{3}, y_{3}\right) \\
\frac{4}{3} \frac{\partial f}{\partial y_{1}}\left(t_{1}, y_{1}\right) & \frac{1}{3} \frac{\partial f}{\partial y_{2}}\left(t_{2}, y_{2}\right) & 0 \\
\frac{9}{8} \frac{\partial f}{\partial y_{1}}\left(t_{1}, y_{1}\right) & \frac{9}{8} \frac{\partial f}{\partial y_{2}}\left(t_{2}, y_{2}\right) & \frac{3}{8} \frac{\partial f}{\partial y_{3}}\left(t_{3}, y_{3}\right)
\end{array}\right)
$$

Due to the continuity of $\frac{\partial f}{\partial y}$, the entries of $S$ are bounded when $h$ and $\boldsymbol{y}$ are restricted to bounded sets. When $h$ is close to zero, the vector $\left(t_{1}, t_{2}, t_{3}\right)$ is close to $\left(t_{0}, t_{0}, t_{0}\right)$, and $W_{h}(\boldsymbol{y})$ is close to $\boldsymbol{y}_{0}=\left(y_{0}, y_{0}, y_{0}\right)$. By continuity of $W_{h}$ it can be seen that if $\bar{B}_{1}\left(\boldsymbol{y}_{0}\right)$ is the closed ball of radius 1 centered at $\boldsymbol{y}_{0}$, then there exists $\epsilon>0$ such that if $0<h<\epsilon$, then $W_{h}\left(\bar{B}_{1}\left(\boldsymbol{y}_{0}\right)\right) \subset \bar{B}_{1}\left(\boldsymbol{y}_{0}\right)$. Moreover, by the continuity of $\frac{\partial f}{\partial y}$, the Frobenius norm of $W_{h}^{\prime}(\boldsymbol{y})$ is strictly less than $1 / 2$ for all $\boldsymbol{y} \in \bar{B}_{1}\left(\boldsymbol{y}_{0}\right)$. Therefore $W_{h}$ is a contraction map (see [5]), which implies that if $0<h<\epsilon$ there exists $\boldsymbol{y} \in \mathbb{R}^{3}$ such that $W_{h}(\boldsymbol{y})=\boldsymbol{y}$. It follows that there is $\boldsymbol{y} \in \mathbb{R}^{3}$ such that $\boldsymbol{y}-W_{h}(\boldsymbol{y})=\mathbf{0}$. This proves that the system of equations (12)-(14) or equivalently, the system (8)-(10) has a unique solution for $h$ sufficiently small.

Equations (8)-(10) can be solved numerically by means of Newton's method using $y_{0}$ as an initial approximation for each of the unknowns $y_{1}, y_{2}, y_{3}$, and using finite differences to approximate the Jacobian matrix (11). 
To build now approximations to $y(t)$ with $t \in\left[t_{3}, t_{3}+T_{1}\right]$, one applies the previous procedure to the regular partition $t_{3}<t_{4}<t_{5}<t_{6}$ using $y_{3}$ as an initail value and $h=T_{1} / 3$ as the distance between consecutive points of the partition. It can be seen that the accuracy of this method is $O\left(h^{4}\right)$ when applied in an interval $\left[t_{0}, t_{0}+T\right]$ using a regular partition with $n+1$ points, and $h=T / n$.

otra manera arbitrario; otro implícito método de (??) se debe resolver preguntar método numéricos

\subsection{A-stability of the method}

The concept of A-stability of a numerical method for initial value problems is useful in the numerical solution of stiff problems (see [1]). The method given by (8)-(10), to which we will refer as Simpson's 3/8, is unconditionally $A$-stable if for any step size $h>0$ the sequence $y_{j}$ produced by the algorithm when applied to the initial value problem $y^{\prime}(t)=-\lambda y(t), y(0)=y_{0}$, satisfies $y_{j} \rightarrow 0$ as $j \rightarrow \infty$ for any complex number $\lambda$ with positive real part. The following results shows the A-stability of the method.

Theorem 2.2 The Simpson's 3/8 method given by (8)-(10) is unconditionally A-stable.

\section{Proof}

Let $h>0$ be any step size, and let $\lambda$ be any complex number with positive real part. Applying Simpson's 3/8 method to the problem $y^{\prime}(t)=-\lambda y(t)$, $y(0)=y_{0}$, we obtain approximations $y_{j}$ to $y(j h)$ satisfying:

$$
\begin{aligned}
y_{2}-y_{0} & =\frac{-\lambda h}{3}\left(y_{0}+4 y_{1}+y_{2}\right) \\
y_{3}-y_{1} & =\frac{-\lambda h}{3}\left(y_{1}+4 y_{2}+y_{3}\right) \\
y_{3}-y_{0} & =\frac{-3 \lambda h}{8}\left(y_{0}+3 y_{1}+3 y_{2}+y_{3}\right) .
\end{aligned}
$$

Solving the system for $y_{1}, y_{2}, y_{3}$, and defining $z=\lambda h=x+i y$, we obtain

$$
\begin{aligned}
& y_{1}=y_{0}\left(\frac{-z^{2}+6 z-z^{3}+12}{3 z^{3}+18 z+11 z^{2}+12}\right)=y_{0} A_{z}, \\
& y_{2}=y_{0}\left(\frac{-z^{2}-6 z+z^{3}+12}{3 z^{3}+18 z+11 z^{2}+12}\right)=y_{0} B_{z}, \\
& y_{3}=y_{0}\left(\frac{-3 z^{3}+11 z^{2}-18 z+12}{3 z^{3}+18 z+11 z^{2}+12}\right)=y_{0} C_{z} .
\end{aligned}
$$


Therefore we obtain that for $j=0,1,2,3, \ldots$,

$$
\begin{aligned}
& y_{3 j+1}=y_{0} A_{z}\left(C_{z}\right)^{j}, \\
& y_{3 j+2}=y_{0} B_{z}\left(C_{z}\right)^{j}, \\
& y_{3 j+3}=y_{0}\left(C_{z}\right)^{j+1} .
\end{aligned}
$$

Hence the method is unconditionally $A$-stable if and only if $\left|C_{z}\right|<1$ for all complex number $z=x+i y$ with positive real part. The condition $\left|C_{z}\right|<1$ is equivalent to

$$
\frac{r_{1}+r_{2}}{r_{3}+r_{4}}<1
$$

where

$$
\begin{aligned}
& r_{1}=\left(3 x^{3}-9 x y^{2}-11 x^{2}+11 y^{2}+18 x-12\right)^{2}, \\
& r_{2}=\left(9 x^{2} y-3 y^{3}-22 x y+18 y\right)^{2}, \\
& r_{3}=\left(3 x^{3}-9 x y^{2}+11 x^{2}-11 y^{2}+18 x+12\right)^{2}, \\
& r_{4}=\left(9 x^{2} y-3 y^{3}+22 x y+18 y\right)^{2} .
\end{aligned}
$$

A calculation shows that

$$
r_{1}+r_{2}-r_{3}-r_{4}=-864 x-936 x^{3}-360 x y^{2}-264 x^{3} y^{2}-132 x y^{4}-132 x^{5},
$$

which is always negative for all $y \in \mathbb{R}$ if $x>0$. This implies that

$$
0<r_{1}+r_{2}<r_{3}+r_{4} \text {, for all } x>0 \text {. }
$$

Therefore $\frac{r_{1}+r_{2}}{r_{3}+r_{4}}<1$ for all $z=x+i y$ with $x>0$. This shows that the method is unconditionally $A$-stable.

\section{$3 \quad$ Numerical experiments}

To test the performance of Simpson's 3/8 algorithm (8)-(10), we have compared the method with the classical 4th-order implicit Runge-Kutta method based on the Gauss-Legendre quadratures (IRKGL4) (see [1]). We used Newton's method to solve the algebraic system of equations, and the Jacobian matrices were approximated by means of finite differences. A drawback of our algorithm is that the number of steps $n$ is required to be of the form $n=3 m$ with $m$ integer. In general we have observed that IRKGL4 gives approximately one more significant digit of accuracy than our algorithm when we use the same number of step size $h$. On the other hand, for the same number of steps, IRKGL4 requires more evaluations of the function $f$. When we tested both 
algorithms using approximately the same number of function evaluations, the accuracy of our algorithm is slightly better than IRKGL4.

Example 1. Consider the problem $y^{\prime}(t)=-100 y+101 e^{t}, t \in[0,1]$, with $y(0)=99 / 100$. The exact solution of this equation is $y(t)=-\frac{e^{-100 t}}{1000}+e^{t}$. Table (1) shows the results of both algorithms when we use the same number $n$ of steps. The step size in this case is $h=1 / n$. The accuracy of the algorithms was calculated with the relative error using the exact solution and the euclidian norm: Error $=\frac{\sqrt{\sum_{j=0}^{n}\left|y_{j}-y(j h)\right|^{2}}}{\sqrt{\sum_{j=0}^{n}|y(j h)|^{2}}}$.

Observe that IRKGL4 yields one more digit of accuracy than the method of Simpson's 3/8. On the other hand, the number of function evaluations (FE) is higher in IRKGL4. Table (2) shows the results of both algorithms when they use approximately the same number of function evaluations. In this case the accuracy of Simpson's 3/8 is higher than IRKGL4.

\begin{tabular}{|l|c|c|c|c|}
\hline$n$ & Error, IRKGL4 & Error, Simpson's 3/8 & FE, IRKGL4 & FE, Simpson's 3/8 \\
\hline 30 & $3.67 \times 10^{-5}$ & $1.61 \times 10^{-4}$ & 300 & 180 \\
\hline 120 & $1.91 \times 10^{-7}$ & $1.76 \times 10^{-6}$ & 1200 & 480 \\
\hline 300 & $4.81 \times 10^{-9}$ & $4.44 \times 10^{-8}$ & 3000 & 1200 \\
\hline
\end{tabular}

Table 1: Comparison between Simpson's 3/8 method and the IRKGL4 method corresponding to Example 1. Here $n$ is the number of steps on $[0,1], \mathrm{FE}=$ number of function evaluations, the error was calculated using the exact solution and the euclidian norm. In this example IRKGL4 has an error smaller than Simpson's 3/8 by approximately a factor of 10. The number of FE is higher in IRKGL4.

\begin{tabular}{|l|c|c|c|c|c|}
\hline FE,IRKGL4 & $n$-IRKGL4 & Error, IRKGL4 & FE, S3/8 & $n$-S3/8 & Error, S3/8 \\
\hline 300 & 30 & $3.67 \times 10^{-5}$ & 300 & 25 & $1.03 \times 10^{-5}$ \\
\hline 1200 & 120 & $1.91 \times 10^{-7}$ & 1200 & 100 & $4.44 \times 10^{-8}$ \\
\hline 3000 & 300 & $4.81 \times 10^{-9}$ & 3000 & 250 & $1.1 \times 10^{-9}$ \\
\hline
\end{tabular}

Table 2: Comparison between Simpson's 3/8 method (abbreviated in this table as S3/8) and the IRKGL4 method based on the number of function evaluations FE in Example 1. Here $n$-IRKGL4 is the number of steps on $[0,1]$ for the IRKGL4 method. In this case the error in Simpson's 3/8 is smaller than in IRKGL4.

Example 2 (Taken from [7]). Consider the problem $y^{\prime}(t)=-200 t y^{2}$, $t \in[-1,0]$, with $y(-1)=1 / 101$. The exact solution of this equation is $y(t)=$ $\frac{1}{1+100 t^{2}}$. The results are presented in Tables (3) and (4). 


\begin{tabular}{|l|c|c|c|c|}
\hline$n$ & Error, IRKGL4 & Error, Simpson's 3/8 & FE, IRKGL4 & FE, Simpson's 3/8 \\
\hline 60 & $2.01 \times 10^{-5}$ & $2.33 \times 10^{-4}$ & 892 & 486 \\
\hline 120 & $1.26 \times 10^{-6}$ & $1.15 \times 10^{-5}$ & 1680 & 954 \\
\hline 600 & $2.01 \times 10^{-9}$ & $1.70 \times 10^{-8}$ & 8384 & 3600 \\
\hline
\end{tabular}

Table 3: Comparison between Simpson's 3/8 method and the IRKGL4 method corresponding to Example 2. Here $n$ is the number of steps on [-1,0]. Again, in this example IRKGL4 has an error smaller than Simpson's $3 / 8$ by approximately a factor of 10 . The number of FE is higher in IRKGL4.

\begin{tabular}{|l|c|c|c|c|c|}
\hline FE,IRKGL4 & $n$-IRKGL4 & Error, IRKGL4 & FE, S3/8 & $n$-S3/8 & Error, S3/8 \\
\hline 892 & 60 & $2.01 \times 10^{-5}$ & 882 & 111 & $1.59 \times 10^{-5}$ \\
\hline 1680 & 120 & $1.26 \times 10^{-6}$ & 1674 & 276 & $3.96 \times 10^{-7}$ \\
\hline 8384 & 600 & $2.01 \times 10^{-9}$ & 8268 & 1380 & $6.15 \times 10^{-10}$ \\
\hline
\end{tabular}

Table 4: Comparison between Simpson's 3/8 method (S3/8) and the IRKGL4 method based on the number of function evaluations FE in Example 2. Here $n$-IRKGL4 is the number of steps on [-1,0] for the IRKGL4 method. As in Example 1, the error in Simpson's $3 / 8$ is smaller than in IRKGL4.

\section{Conclusions}

We have proposed a 4th-order unconditionally A-stable method based on Simpson's rule to approximate the solution of initial value problems. The competitiveness of the method is shown by comparing it with another 4th-order unconditionally A-stable method, the implicit Runge-Kutta method based on the Gauss-Legendre quadratures (IRKGL4). Although the latter method gives approximation errors smaller than our method by a factor of 10 when they use the same number of steps, our method uses less function evaluations. Testing both algorithms in terms of function evaluations results in a smaller error of our algorithm than the on in IRKGL4. A drawback of our algorithm is that the number of steps $n$ is required to be of the form $n=3 m$ with $m$ integer. Numerical results for the vector form of the initial value problem will be reported in the future.

\section{References}

[1] A. Iserles, A first course in the numerical analysis of differential equations, Cambridge University Press (1996).

[2] W. E. Milne, Numerical integration of ordinary differential equations, Amer. Math. Month. 33(1926)5, pp. 455-460.

[3] W. E. Milne, R. R. Reynolds Stability of a numerical solution of differential equations, J. Assoc. Comput. Mach., 6(1959), pp. 196-203. 
[4] W. E. Milne, R. R. Reynolds Stability of a numerical solution of differential equations II, J. Assoc. Comput. Mach., 7(1960), pp. 46-56.

[5] W. Rudin, Principles of Mathematical Analysis, McGraw-Hill (1976).

[6] H. J. Stetter, Stabilizing predictors for weakly unstable correctors, Math. Comp., 19(1965), pp. 84-89

[7] J. Stoer, R. Bulirsch, Introduction to Numerical Analysis, Springer-Verlag (1991).

Received: May 3, 2007 